\title{
Computational Intelligence Methods for Time Series Analysis and Forecasting: Special Issue of IWANN 2017
}

\author{
Olga Valenzuela ${ }^{1} \cdot$ Fernando Rojas $^{2} \cdot$ Ignacio Rojas $^{3}$ \\ Published online: 22 July 2020 \\ (c) Springer Science+Business Media, LLC, part of Springer Nature 2020
}

\section{Preface}

The 14th International Work-Conference on Artificial Neural Networks (IWANN) is a biennial meeting seeks to provide a discussion forum for scientists, engineers, educators and students about the latest discoveries and realizations in the foundations, theory, models and applications of systems inspired on nature, using computational intelligence methodologies, as well as in emerging areas related to the above items. As in previous editions, we strongly emphasize the wide range of topics comprised under the umbrella of IWANN2017 and, in particular, we focus on trending topics such as Deep Learning, Human-centred Computing and developments encompassed in and related to the Human Brain Project.

This approach will be both theoretical and practical, through invited talks, tutorials, workshops, presentation of demos and functional prototypes. Besides, we offer the possibility to carry out a limited number of virtual presentations for those who want to participate but they have serious difficulties on travelling.

Since the first edition in Granada (LNCS 540, 1991), the conference has evolved and matured, and most of the topics involved have achieved a maturity and reinforced consolidation. The fourteenth edition of the IWANN conference "International Work-Conference on Artificial Neural Networks" was held in Cadiz, (Spain) during June 14-16, 2017. The list of topics in the successive Call for Papers has also evolved, resulting in the following list for the present edition:

\section{Ignacio Rojas}

irojas@ugr.es

Olga Valenzuela

olgavc@ugr.es

Fernando Rojas

frojas@ugr.es

1 Department of Applied Mathematics, University of Granada, 18071 Granada, Spain

2 Department of Computer Architecture and Computer Technology, University of Granada, 18071 Granada, Spain

3 Information and Communications Technology Centre (CITIC-UGR), University of Granada, 18071 Granada, Spain 
1. Mathematical and theoretical methods in computational intelligence Mathematics for neural networks. RBF structures. Self-organizing networks and methods. Support vector machines and kernel methods. Fuzzy logic. Evolutionary and genetic algorithms.

2. Neurocomputational formulations Single-neuron modelling. Perceptual modelling. System-level neural modelling. Spiking neurons. Models of biological learning.

3. Learning and adaptation Adaptive systems. Imitation learning. Reconfigurable systems. Supervised, non-supervised, reinforcement and statistical algorithms.

4. Emulation of cognitive functions Decision Making. Multi-agent systems. Sensor mesh. Natural language. Pattern recognition. Perceptual and motor functions (visual, auditory, tactile, virtual reality, etc.). Robotics. Planning motor control.

5. Bio-inspired systems and neuro-engineering Embedded intelligent systems. Evolvable computing. Evolving hardware. Microelectronics for neural, fuzzy and bioinspired systems. Neural prostheses. Retinomorphic systems. Brain-computer interfaces (BCI) Nanosystems. Nanocognitive systems.

6. Advanced topics in computational intelligence Intelligent networks. Knowledgeintensive problem solving techniques. Multi-sensor data fusion using computational intelligence. Search and meta-heuristics. Soft Computing. Neuro-fuzzy systems. Neuroevolutionary systems. Neuro-swarm. Hybridization with novel computing paradigms.

7. Applications Expert Systems. Image and Signal Processing. Ambient intelligence. Biomimetic applications. System identification, process control, and manufacturing. Computational Biology and Bioinformatics. Parallel and Distributed Computing. Human Computer Interaction, Internet Modeling, Communication and Networking. Intelligent Systems in Education. Human-Robot Interaction. Multi-Agent Systems. Time series analysis and prediction. Data mining and knowledge discovery.

At the end of the submission process of IWANN 2017, and after a careful peer review and evaluation process (each submission was reviewed by at least 2, and on the average 2.8, program committee members or additional reviewers), 126 papers were accepted for oral or poster presentation, according to the recommendations of reviewers and the authors' preferences.

High-quality candidate papers (11 contributions) were invited to submit an extended version of their conference paper to be considered for special publication in this issue of Neural Processing Letters related with Time Series. These authors were selected after the recommendation of the reviewers of the conference papers, the opinion of the chairs of the different sessions and the guest editors. At least three independent and anonymous experts again carefully reviewed the extended versions and finally 7 papers were selected as appropriate for publication. In the present issue of Neural Processing Letters, it is a pleasure to present you these contributions that provide a clear overview of the thematic areas covered by the IWANN conference, ranging from theoretical aspects to real-world applications of natureinspired system.

The first paper, "Application of Quantile Graphs to the Automated Analysis of EEG Signals" by Andriana Campanharo et al., is focused in the evaluation of the "quantile graph" method (QG) under a wide range of experimental conditions of electroencephalogram (EEG). The database of EEG contains time series that correspond to five conditions: (1) EEG recordings of healthy (volunteers with eyes open) (2) EEG recordings of healthy (volunteers with eyes closed), (3)intracranial EEG signals from epileptic patients that were recorded during seizure-free intervals in the epileptogenic zone, (4) intracranial EEG signals from epileptic patients that were recorded in the hippocampal formation of the opposite hemisphere of the brain and (5) EEG recordings of seizure activity, which were selected from sites that 
were exhibiting ictal activity. The results presented in this contribution, have shown that the QG method can differentiate epileptic from normal data and also distinguish the different abnormal stages/patterns.

In the paper entitled "Fuzzy Time Series Models Using Pliant- and Asymptotically Pliant Arithmetic-based Inference", by Tamas Jonas et al., the main goal is to analyze the problem of fuzzy arithmetics-based fuzzy time series modeling. In this contribution, the fuzzy output is a so-called either pliant or quasi pliant (q-pliant) number. The novelty of the proposed fuzzy inference methodology is mainly based in the fact that its fuzzy output is obtained by fuzzy arithmetic operations (weighted summation of pliant numbers or q-pliant numbers), which are the consequents of the fuzzy rules. Experimental results are provided to illustrate the effectiveness of the proposed method in real examples, and compared with other well-known time series methodologies.

Manuel Dorado-Moreno et al., in the contribution entitled "Ordinal multi-class architecture for predicting wind power ramp events based on reservoir computing" present an novel contribution in the field of using of an Echo State Network (ESN) based learning model to approach a problem consisting in predicting wind power ramp events, applied to a real case-study from wind farms in Spain. In this paper, the authors face the wind power ramp events (WPRE) prediction, presenting a novel methodology, by considering a multi-class classification problem with three different classes of WPREs: negative ramps, non-ramps and positive ramps. The proposed method is compared with other classical classifiers, such as Support Vector Machines or nominal logistic regression, showing robust and accuracy performance.

The paper entitled "A Multi-resolution Approximation for Time Series ", by Heider Sanchez et al., presents a multi-resolution representation for time series (MTVA) based on the local trends and mean values, which integrates the advantage of the multi-resolution techniques and the local trends on each level of resolution. In this contribution, extensive experimental results are provided to demonstrate the effectiveness of the proposed method.

The paper, presented by Alejandro Catalina Feliú et al., "Machine Learning Nowcasting of PV Energy using Satellite Data" proposes a new technique for the exploitation of satellite-based information, which has been widely used to nowcast solar irradiance values and photovoltaic (PV) energy productions, together with clear sky irradiance estimates for the nowcasting of PV energy productions over peninsular Spain, using different machine learning models. In this contribution, prediction horizons of up to six hours has been used, with Gaussian Support Vector Regression (SVR) being statistically better than the other models at each horizon. Although linear models give weaker results, the methodology will take advantage of the spatial sparsity provided by Lasso to try to identify the concrete areas with a larger influence on PV energy nowcasts.

Argimiro Arratia et al., in the contribution entitled "An Evaluation of Equity Premium Prediction using Multiple Kernel Learning with Financial Features" present a non linear modeling for predicting of Equity Premium. They focused on the Welch and Goyal work, showing that several variables proposed in the finance literature are of no use as exogenous information to predict the equity premium under linear regressions. In this contribution different experimental results have been presented to show the improvement of the proposed methodology.

Finally, the last selected paper entitled: “Time Series, Spectral Densities and Robust Functional Clustering " by D. Rivera-Garcia et al., presents a clustering algorithm for stationary time series based on the use of estimated spectral densities,(measured as functional data), as the basic characteristic of stationary time series for clustering purposes. The presented 
methodology is analyzed in a simulation study, and real data example shows that the proposed robust functional clustering method (RFC) for time series clustering has a good performance.

The Guest Editors would like to express their gratitude to all the people who supported them in the compilation of this special issue, and specially to the contributing authors for their submissions and to the anonymous reviewers for their comments and useful suggestions in order to improve the quality of the papers.

They would also like to express their gratitude to Editors-in-Chief M. Verleysen and M.H. Hassoun, for providing us with the opportunity to publish this set of selected papers in the present issue. It is a pleasure for us to invite all authors and interested readers of this issue to future IWANN conferences, which will be announced at http://iwann.ugr.es.

Publisher's Note Springer Nature remains neutral with regard to jurisdictional claims in published maps and institutional affiliations. 